\title{
Research on 5G Miniaturized Quasi-Yagi Antenna
}

\author{
Meng Li \\ School of \\ Communication \\ Engineering,
}

Chengdu University of

Information Technology

Chengdu, China

\author{
QiuQiang Luo \\ School of \\ Communication \\ Engineering,
}

Chengdu University of

Information Technology

Chengdu, China

\author{
Hao Zou \\ School of \\ Communication \\ Engineering, \\ Chengdu University of \\ Information Technology \\ Chengdu, China
}

\author{
Jiaming Xia \\ School of \\ Communication \\ Engineering, \\ Chengdu University of \\ Information Technology \\ Chengdu, China
}

\begin{abstract}
This paper proposes an improved miniaturized quasi-Yagi antenna that works in the 5G millimeter wave band. Compared with the traditional quasi-Yagi antenna, reasonable use of the layout can reduce the longitudinal space of the antenna, greatly reducing the size of the antenna to a size of $15 \mathrm{~mm} \times 26 \mathrm{~mm} \times 1 \mathrm{~mm}$., This antenna achieves a measured $43.8 \%(23.7-37 \mathrm{GHz})$ frequency bandwidth for voltage standing-wave ratio 2 has a wider bandwidth. The maximum measured gain of the antenna is $7.41 \mathrm{dBi}$ at $29 \mathrm{GHz}$ and yhe minimum is $4.44 \mathrm{dBi}$ at $32 \mathrm{GHz}$. Meet the efficiency requirements of $5 \mathrm{G}$ millimeter wave mobile communication systems

Keywords: millimeter wave; Miniaturized; Microstrip; quasi-Yagi antenna; Broadband.
\end{abstract}

\section{INTRODUCTION}

The position of the antenna in the entire radio communication system is very important, quality is directly affecting the distance and communication effect of the transmission and reception distance. It can be said that there is no radio communication without the antenna. As a kind of antenna with simple structure and good orientation, Yagi antenna has been studied by many experts and scholars at home and abroad, and is also widely used in various communication systems[1-2]. However, the traditional Yagi antenna is bulky and costly. Microstrip antennas are widely used due to their small size, light weight, simple manufacturing, and easy conformality. With the development of microstrip antenna technology, the design principle of Yagi antenna is combined with microstrip technology, which is called "quasi-yagi antenna"[3]. Y. Qian et al. first applied microstrip technology to Yagi antenna design [3]. Therefore, the miniaturization of Yagi antenna has become a hot topic in today's society. The bow-tie antenna is considered to be the starting point for the development of a miniaturized model with similar characteristics [4-5]. Mr tang of Huaqiao University and others have made the substrate size of the antenna only only $1 / 3$ of the free wavelength of the center frequency through reasonable layout and glory loading technology, and relative bandwidth is $42.6 \%$, but the gain is only $4 \mathrm{dBi}$. Based on this research, this paper designs a miniaturized quasi-Yagi antenna that works in the 5G high frequency band to achieve extremely wide bandwidth ( $48 \%$ for VSWR 2 measurement).

\section{QUAI-YAGI DESIGN}

Figure 1 shows the general shape of a ternary quasi-yagi antenna. The antenna consists of a port, a $50 \Omega$ feed line, an impedance transformer, a balun transformer, a director, and a reflector.

As can be seen from the figure 1, the space under the unused portion of the conventional quasi-Yagi antenna is too large. Therefore, we improve the antenna according to the rational layout and capacitive loading technique in [6]. Design the shape of the director to resemble a bow shape, slightly modifying the shape of the balun transformer。The designed antenna is shown in figure 2 .

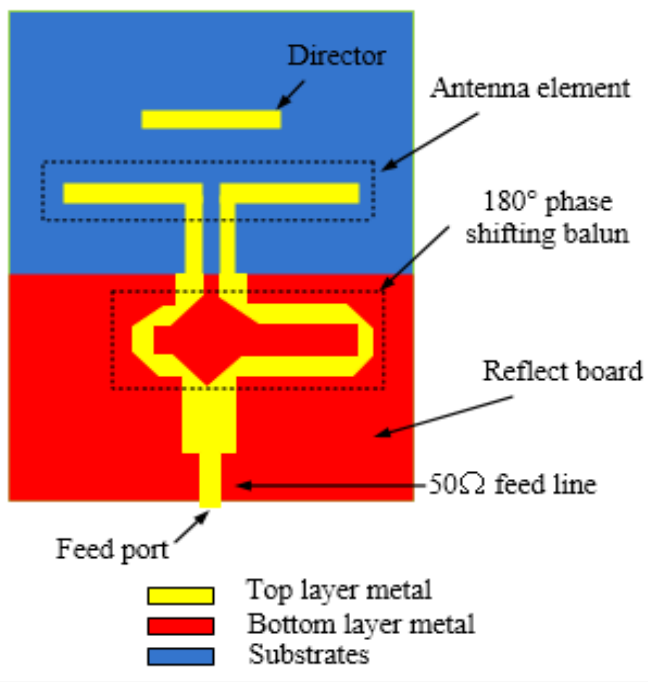

Figure. 1. traditional of the quasi-Yagi antenna

Figure 2 shows the layout of the designed quasi-yagi antenna. The antenna is fabricated on a substrate of material FR4 (substrate thickness $\mathrm{h}$ is $1 \mathrm{~mm}$ ). The bottom consists of a "U"-shaped copper plate. The top-level quasi-yagi antenna passes through a reasonable layout, and the distribution is more concentrated than the traditional quasi-yagi antenna in Figure 1. The backside conductor of the substrate designed to be "U" shaped serves as both the ground for the microstrip line circuits (ports, feeders, impedance transformers and baluns) and also acts as a reflector to the antenna. There is a big breakthrough in the traditional rectangular bottom plate.

The design of the antenna in figure 2 maintains the use of a truncated ground plane as the reflector element. The driven printed dipole is used to generate $T E_{0}$ surface waves with very small undesired $T M_{0}$ content [6]. In this optimized quasi-Yagi design, the antenna director components are 


\begin{tabular}{|l|r|l|l|l|r|}
\hline variable & length & variable & length & variable & length \\
\hline $\mathrm{W}$ & 7.125 & $\mathrm{fl} 1$ & 6.2 & $\mathrm{fs} 1$ & 2.7 \\
\hline $\mathrm{L}$ & 14.25 & $\mathrm{fl} 2$ & 9.4 & $\mathrm{fs} 2$ & 4 \\
\hline $\mathrm{fw} 1$ & 1.8 & $\mathrm{fl} 3$ & 2.4 & $\mathrm{fs} 3$ & 4.425 \\
\hline $\mathrm{fw} 2$ & 2.7 & $\mathrm{fl} 4$ & 3.2 & $\mathrm{rl}$ & 6.1 \\
\hline $\mathrm{fw} 3$ & 1.8 & $\mathrm{bl} 1$ & 11.2 & $\mathrm{rl}$ & 6.1 \\
\hline $\mathrm{fw} 4$ & 3.2 & $\mathrm{bl}$ & 3.6 & $\mathrm{bw}$ & 1.8 \\
\hline $\mathrm{fw} 5$ & 3 & $\mathrm{fd}$ & 2.2 & $\mathrm{rw}$ & 1.8 \\
\hline
\end{tabular}

Table 1. Antenna parameter table (unit:mm)

shorter than conventional quasi-Yagi antenna designs, contributing to a wide range of antenna band characteristics.

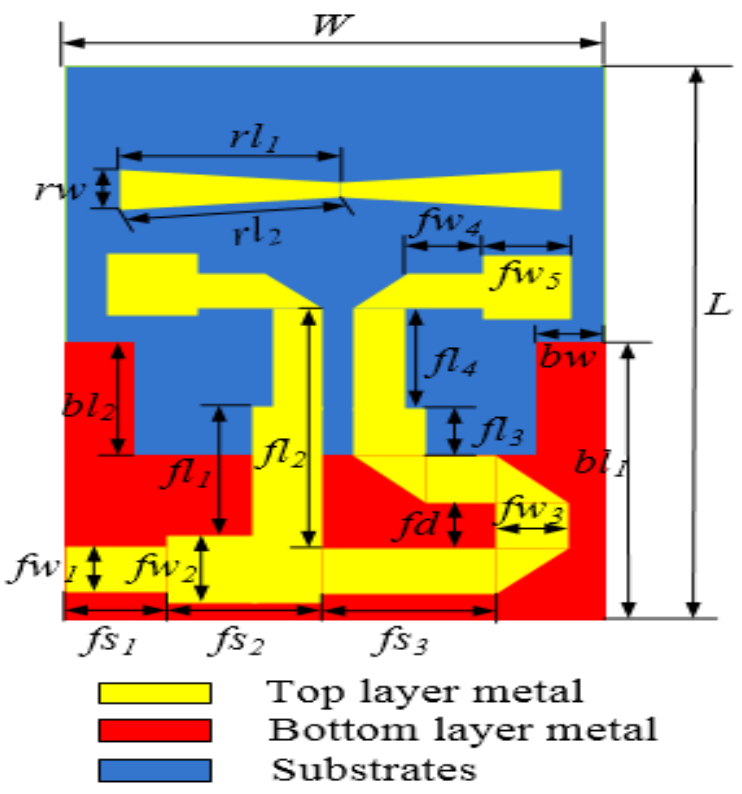

Figure.2.Schematic of the quasi-Yagi antenna.

\section{SIMULATIONS}

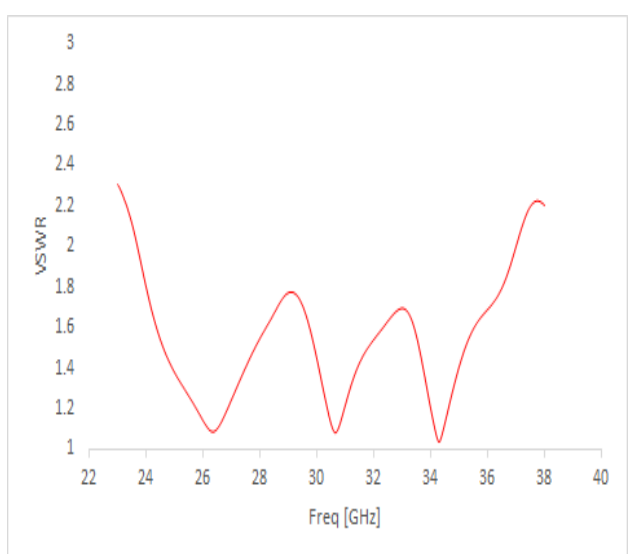

Figure.3. Voltage standing wave ratio

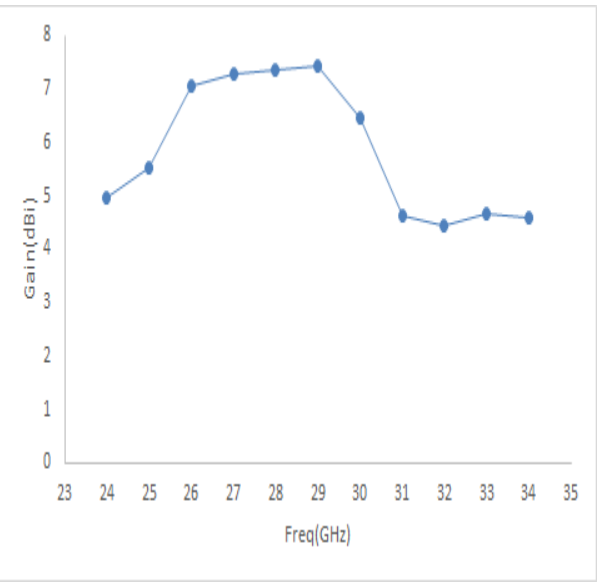

Figure.4. Gain of Antenna

It can be seen from the figure 3 and figure 4 that we have achieved extremely broad bandwidth (measured $48 \%$ for VSWR 2) in 23.7-37-34 GHz in the 5G FR2 band, which meets the requirements in the millimeter wave band. The maximum measured gain of the antenna is $7.41 \mathrm{dBi}$, which is $3.5 \mathrm{dBi}$ higher than the gain in general literature, and the size is only $15 \mathrm{~mm} \times 26 \mathrm{~mm} \times 1 \mathrm{~mm}$, which realizes the miniaturization of the quasi-Yagi antenna.

\section{CONCLUSION}

This paper proposes a miniaturized quasi-Yagi antenna, which saves space by changing the layout of the antenna and the change of the shape of director and the balun transformer. The size of the antenna is $15 \mathrm{~mm} \times 26 \mathrm{~mm} \times 1 \mathrm{~mm}$. The director is designed as a bow shape, which expands the bandwidth of the antenna. This antenna achieves a measured $43.8 \%$ (23.7$37 \mathrm{GHz}$ ) frequency bandwidth for voltage standing-wave ratio 2 , and have a wider bandwidth. In the same kind of literatures, the antenna size is smaller and the gain is higher, and can be used in 5G mobile communication systems and other wireless communication systems in the frequency band, and has high engineering

value. 


\section{REFERENCES}

[1] Wang, Hao , et al. "Design of a wideband planar microstrip-fed quasi-Yagi antenna." Progress in Electromagnetics Research Letters 46.46(2014):19-24.

[2] Floch, Jean Marie . "Broadband Quasi-Yagi Antenna for WiFi and WiMax Applications." Wireless Engineering and Technology 04.2(2013):87-91.

[3]Qian, Yongxi , et al. "A uniplanar quasi-Yagi antenna with wide bandwidth and low mutual coupling characteristics." IEEE Antennas and Propagation Society International Symposium. 1999 Digest. Held in conjunction with: USNC/URSI National Radio Science Meeting (Cat. No.99CH37010) IEEE, 2002.

[4]Kaneda, Noriaki, Y. Qian, and T. Itoh . "A Novel YagiUda Dipole Array Fed by A Microstrip-To-CPS Transition." Asia Pacific Microwave Conf Dig 1998.

[5]Deal, W. R. , et al. "A new quasi-Yagi antenna for planar active antenna arrays." IEEE Transactions on Microwave Theory and Techniques 48.6(2000):910-918.

[6] Qian, Y, et al. "Mutual coupling and mitigation in twodimensional phased arraysbased on planar quasi-Yagi antennas." Asia-pacific Microwave Conference IEEE, 2000. 